\title{
Inklusion durch Wohlfahrtsmix
}

\section{Menschen mit Behinderung brauchen eine individuelle Mischung verschiedener Unterstützungsleistungen}

Cordula Barth

Cordula Barth ist Diplom-Gerontologin und Diplom-Sozialpädagogin mit Berufserfahrung in der Behinderten- und Jugendhilfe sowie der Alten- und Entwicklungszusammenarbeit. Nach einer wissenschaftlichen Tätigkeit in einem vom Bundesministerium für Arbeit und Soziales geförderten Forschungsprojekt zur Gestaltung von Versorgungsstrukturen für alt gewordene Menschen mit Behinderung ist sie seit 2010 am Zentrum für Planung und Evaluation Sozialer Dienste (ZPE) der Universität Siegen im Team beschäftigt, das mit der Evaluation des Projekts »Personenzentrierte Steuerung der Eingliederungshilfe in Hessen « (PerSEH) betraut ist.

E-Mail barthcordula@hotmail.com
Unter Wohlfahrtsmix versteht man vor allem das Zusammenspiel von Staat, Markt, Verbänden und anderen Anbietern von Dienstleistungen sowie den Leistungsempfängern und ibrem persönlichen Umfeld. Dieses Konzept kann insbesondere bei Menschen mit einer Behinderung $z u$ Arrangements beitragen, die dem Unterstützungsbedarf des jeweiligen Individuums besser gerecht werden kann als fertige Dienstleistungsangebote.

Die UN-Konvention über die Rechte von Menschen mit Behinderungen wurde 2009 von Deutschland ratifiziert und ist seither geltendes Recht. Diese Konvention fokussiert die allgemeinen Menschenrechte auf die Perspektive von Menschen mit Behinderung und verfolgt das Leitbild der Inklusion.

Danach soll die Gesellschaft in ihren Strukturen so gestaltet sein, dass alle Menschen unabhängig von Alter, Geschlecht oder Behinderung in ihrer Individualität wertgeschätzt und nicht ausgegrenzt werden. Alle sollen in den verschiedenen Lebensbereichen wie beispielsweise Bildung, Beruf und Wohnen gleichberechtigt und selbstbestimmt an der Gesellschaft teilhaben. Voraussetzung dafür ist sowohl eine passgenaue individuelle Unterstützung sowie die Zugänglichkeit von allgemeinen Diensten und die Überwindung von Barrieren. Zudem dürfen Hilfen und Dienstleistungen aufgrund ihrer Spezialisierung segregierenden Charakter haben und somit ebenfalls Hindernisse im Gemeinwesen darstellen.

Diesem Leitgedanken folgend sollten Menschen mit Behinderung durchlässige und flexible Hilfesysteme und Angebotsstrukturen zur Verfügung stehen, die zu nahtlos ineinander greifenden Unterstützungsarrangements führen. Dabei geht es um den Auf- und Ausbau einer adäquaten Nahversorgung und Infrastruktur durch eine Angebots- und örtliche Teilhabeplanung im Rahmen der kommunalen Daseinsvorsorge.
Kooperationen und Vernetzungen bieten sich als zentrale Strategie an, so dass eine inklusive und umfassende Versorgung gewährleistet wird und kostenintensive, nicht der Wahlfreiheit der Nutzerinnen und Nutzer dienende Doppelstrukturen vermieden werden können.

Die Passgenauigkeit von am individuellen Bedarf orientierten Angeboten wird häufig mit dem Konzept eines Wohlfahrtsmix in Verbindung gebracht. Bei diesem Konzept geht es um das $\mathrm{Zu}$ sammenspiel von Staat, Markt, Verbänden und anderen Anbietern von Dienstleistungen sowie den Leistungsempfängern und ihren persönlichen Bezügen. Dabei hat der Wohlfahrtsmix in zweierlei Hinsicht positive Auswirkungen: sowohl in ökonomischer Hinsicht durch eine optimale Nutzung vorhandener Ressourcen wie auch in politischer Hinsicht durch partizipative Elemente des Empowerments von Leistungsbeziehern im Sinne einer gemeinsamen Steuerungsverantwortung (vgl. Evers 2011: 265-273).

\section{Gewachsene Strukturen behindern personenorientierte Teilhabeleistungen}

Es bedarf neben einer örtlichen Teilhabeplanung geeigneter Assessment- und Planungsinstrumente für personenzentrierte Unterstützungs- oder Teilhabeleistungen. Für Leistungsträger wie auch Leistungserbringer ist bei der Ausgestaltung von Leistungen allerdings oft nicht der Grundgedanke der Inklusion leitend, sondern historisch gewachsene Strukturen des Zusammenwirkens und ein sich daraus ergebendes - und nicht zuletzt durch die Sozialgesetzgebung manifestiertes segregiertes System von Zuständigkeiten und Zielgruppen, das häufig zu nur mangelhaft koordinierten Leistungsarrangements oder Versorgungslücken führt.

Die sich hieraus ergebende Unübersichtlichkeit wird noch dadurch verstärkt, 
dass die »Gesetzgebenden ... eine quasistaatliche Ebene an Richtlinien, Empfehlungen und Rahmenverträgen aushandeln [lassen.] ... Oft sind diese Festlegungen und Ausgestaltungen sehr kompliziert, ihr Zustandekommen ... langwierig, die Verhandlungsprozesse selbst nicht transparent. Und sie sind selten mehr als Minimalkonsense. " (Frevel et al. 2008: 143). Zudem »vertreten die Verbände Personen und Einrichtungen, die ihrerseits nicht nur fachliche, sondern in erster Linie wirtschaftliche Interessen haben." (ebd.: 144).
Das Projekt wurde in zwei Landkreisen erprobt und basiert auf drei Säulen: Es wurde ein neues Instrument zur Planung und Überprüfung der Teilhabeleistungen für (erwachsene) Menschen mit Behinderung in Verbindung mit einem zielgruppenspezifischen Beratungsverfahren über die Qualität und Passgenauigkeit der Hilfen in einer sogenannten Hilfeplankonferenz eingeführt. Zeitgleich wurde die Finanzierung von einer pauschalierten Finanzierung der Maßnahmen auf ein zeitbasiertes Finanzierungssystem umgestellt (vgl. Kunze et al. 2008).

\section{»Nach wie vor wird zunächst nach reinem Platz<in einem bestehenden Angebot gesucht «}

So hemmen historisch gewachsene Denk- und Handlungsmuster beispielsweise eine adäquate Umsetzung der Priorität ambulanter vor stationärer Versorgung oder des Subsidiaritätsprinzips im Sinne eines Vorrangs von Hilfen einer untergeordneten Ebene, also der selbst und im Umfeld geleisteten Unterstützung vor staatlichen Hilfen. Verschärft wird die Situation dann, wenn die Kommune als Ort der Daseinsvorsorge »knapp bei Kasse ist « - was auf die meisten Kommunen zutrifft. Meist gewähren Leistungsträger nur Pflichtleistungen; freiwillige, präventiv wirkende Leistungen (wie z. B. viele Beratungsleistungen nach SGB XII) entfallen oder die Angebotspalette in der Region wird nicht gezielt gesteuert, so dass Versorgungssicherheit wie auch eine zur Inklusion bereite Gesellschaft langfristig nur bedingt gewährleistet sind.

Diese strukturellen und gesetzlichen Grenzen werden auch in Projekten zur Weiterentwicklung von Leistungen des Sozialstaates deutlich. Hierzu soll exemplarisch der Bezug zu Ergebnissen der Begleitforschung zu einem Projekt zur Weiterentwicklung der Eingliederungshilfe »Personenzentrierte Steuerung der Eingliederungshilfe in Hessen " (PerSEH) hergestellt werden.
Die einjährige Begleitforschung ergibt neben eindeutig positiven Entwicklungen durch das Projekt, dass sich die bisherigen Veränderungen auf Einzelfälle beschränken und gewisse Beharrungstendenzen bestehender Systeme und Routinen existieren. Diese Tendenzen sind begründet in der Expansion und Ausdifferenzierung der Einrichtungen und Dienste der Behindertenhilfe zu »sicheren Schutzräumen für Menschen mit Behinderung « und gleichzeitig in ihrer Entwicklung zu an Prinzipien der Expansion und Gewinnmaximierung orientierten Sozialunternehmen (vgl. Frevel et al. 2008: 143 f.). So werden der individuelle Bedarf wie auch die Wünsche von Leistungsberechtigten meist auf bestehende Angebotsstrukturen der Eingliederungshilfe bezogen - ein kreatives Entwickeln neuer Teilhabearrangements findet nur selten statt.

Eine Vernetzung von in der Kommune vorhandenen Angeboten, wie beispielsweise Bildungseinrichtungen mit Sozialunternehmen, anstatt einer Erweiterung der segregierenden zielgruppenspezifischen Unterstützungsleistungen, ist bis dato in geringem Maße anzutreffen, auch wenn eine inklusive Sozialraumgestaltung die Maxime für eine effiziente Eingliederungshilfe sein soll.
Genauso wenig werden im Zuge der individuellen Hilfeplanung weder eigene noch Ressourcen im Umfeld systematisch akquiriert oder auf das Ermöglichen einer eigenständigeren und eigenverantwortlichen Lebensführung bezogen. Im Allgemeinen finden Veränderungen im Sinne einer fortschreitenden Ambulantisierung allenfalls im Lebensbereich "Wohnen « statt. Dies zieht allerdings keineswegs die Abkehr von den gängigen Platzierungsmustern in der Behindertenhilfe nach sich: Zunächst muss »ein Platz« in einem ambulanten Angebot frei werden, bevor eine Klientin oder ein Klient mit den entsprechenden Voraussetzungen (meist einem geringeren individuellen Bedarf und einem gewissen $\mathrm{Ma}$ an lebenspraktischen Fähigkeiten) diesen »Platz « besetzen kann (vgl. Rohrmann et al. 2011, 2011a).

Das Konzept des Wohlfahrtsmix geht davon aus, dass Unterstützungsleistungen durch eine Mischung aus personenbezogenen Leistungen und (kommunaler) Angebots- und Sozialplanung organisiert werden. Somit ist die Verknüpfung von Steuerungs- und Planungsansätzen, von Leistungen im Einzelfall und sozialraumorientierter Zusammenarbeit der kommunalen Akteure relevant. Dabei sind und das hat auch die Nutzerbefragung im Rahmen der Evaluation des Projekts »Personenzentrierte Steuerung der Eingliederungshilfe in Hessen « ergeben - der Umgang mit und die Verständlichkeit des Planungsinstruments ausschlaggebend für die Qualität der Leistungen. Die aus der Orientierung an den Wünschen und Zielen der Leistungsberechtigten resultierende Intensität des Gesprächs, wie auch die damit verbundenen Lern- und Emanzipationsprozesse, werden sowohl von Menschen mit Behinderung selbst, wie auch von ihren professionellen Unterstützungspersonen positiv hervorgehoben. Grundlegend dafür sind aufseiten der Leistungsberechtigten die Kenntnis der Auswahlmöglichkeiten und ein größtmögliches Verständnis der sozialstaatlichen Planungs- und Erbringungsstrukturen. Aufseiten der Unterstützungspersonen sollte die Bereitschaft bestehen, bestehende Denk- und Handlungsmuster zu überprüfen und gegebenenfalls zu modifizieren. Nur dann können gemeinsam kreative Wege entwickelt werden, um den Zielen näher zu kommen (vgl. Rohrmann et al. 2011b). 


\section{Beispiel USA: »my plan «}

Es ist ungewöhnlich, wenn bei sozialstaatlichen Steuerungs- und Planungsfragen der Blick nach Westen wandert, allerdings ist es in diesem Fall lohnenswert.

Mitte der 1970er Jahre wurde in den USA die Selbstvertretungsbewegung »People First « von Menschen mit kognitiven Beeinträchtigungen oder mit Lernschwierigkeiten gegründet. Ausschlaggebend war die Notwendigkeit des Aufund Ausbaus einer inklusiven Gesellschaft und der Beendigung, zumindest Reduktion von sozialen Zuschreibungen von Menschen mit Behinderung für ihre gleichberechtigte Teilhabe an der Gesellschaft. Im Rahmen dessen veranstaltet dieses Netzwerk Schulungen, übersetzt und veröffentlicht in »Leichter Sprache « (eine barrierefreie Ausdrucksweise durch kurze Sätze, einfache grammatische Konstruktionen, die Vermeidung von Fremdund Fachwörtern sowie die Verdeutlichung der Kernaussage durch Piktogramme).

People First hat nun sowohl ein Konzept, das sogenannten »person centred planning « und daraus resultierend einen Hilfeplan mit dem Namen »my plan « entwickelt, der Leistungsansprüche für erwachsene Menschen mit Behinderung nach sich zieht. Dieser Plan ist in die vier Kategorien »Gemeinweseneinbindung und Freizeitgestaltung «, »Gesundheit «, »Wohnen «, »Arbeit « und »Persönliche Entwicklung « untergliedert. Dort werden mittels standardisierter, leicht verständlicher Fragen die aktuelle und die anvisierte Lebenslage erhoben. Im Hinblick auf die Zukunftsvision werden konkrete Ziele, Ressourcen und Stärken sowie Bar- rieren und Ängste eruiert. Zudem wird nach den Stärken und Schwächen der leistungsberechtigten Person sowie nach aktuellen und erwünschten Unterstützungspersonen gefragt.

Seit Ende des letzten Jahrtausends gibt es »People First « in Form von »Mensch zuerst - Netzwerk People First Deutschland e. V. "sowie das Konzept der »Persönlichen Zukunftsplanung « auch in Deutschland. Allerdings bildet die "persönliche Zukunftsplanung « meist nur eine Grundlage für die verschiedenen Instrumente zur individuellen Hilfeplanung in Deutschland wie beispielsweise dem Integrierten Teilhabeplan (ITP) oder der Individuellen Hilfeplanung (IHP3).

Was für Perspektiven bringt aber nun eine von der Selbstvertretung entwickelte Planungssystematik? Kann der so notwendige frische und reformerische Wind

\section{Institutionelle Hilfeplanung versus Persönlicher Zukunftsplanung}

Die traditionelle Hilfeplanung in der Behindertenhilfe ist behinderungsorientiert. Dem liegt die Annahme zugrunde, es sei besonders wichtig zu wissen, welche Behinderung jemand hat, um ihm seiner Behinderung entsprechende Bildungs-, Wohn-, Arbeits- und Freizeitangebote zu machen. Persönliche Zukunfts- planung stellt eine neue Form der Planung der Unterstützung für Menschen mit Behinderung dar. Die traditionelle Hilfeplanung in der Behindertenhilfe wird als eine eher institutionelle Hilfeplanung charakterisiert, dem die veränderte Sichtweise einer eher individuellen Hilfeplanung entgegengestellt wird.

\section{Institutionelle Hilfeplanung \\ - Orientierung an Behinderung \\ - Betonung von Defiziten und Bedürfnissen \\ - Ziel: oft Reduzierung von negativen Verhaltensweisen}

- Hilfeplanung abhängig vom professionellen Urteil, oft standardisierte Tests und Begutachtungen

- Schriftliche Berichte

- Sieht die Person im Kontext der verfügbaren Maßnahmen und Behinderteneinrichtungen, dies sind oft Lebensräume speziell für Menschen mit Behinderungen

- Professionelle Distanz durch Betonung der Unterschiede

- Staatlich geregelte Verfahrensweisen, Blickrichtung Kostenträger

- Person ist an der Erstellung der Hilfeplanung (oft nur teilweise) beteiligt

- Zielrichtung: Stärkung und Ausbau der Institution durch Angebot geeigneter Maßnahmen
Persönliche Zukunftsplanung

- Orientierung an der individuellen Person

- Suche nach Fähigkeiten und Stärken

- Ziel: Erweiterung der Lebensqualität

- Hilfeplanung abhängig von der Person, Familie, Freunde und Fachleuten, verlangt mit der Person Zeit zu verbringen, um sie kennen zulernen, und gemeinsam eine gute Beschreibung zu erarbeiten

- »Geschichten«, Episoden von Menschen, die die Person gut kennen

- Sieht die Person im Kontext des regulären Lebens in der Region

- Bringt Menschen zusammen durch die Identifizierung von Gemeinsamkeiten

- Verfahrensweise nicht vorgeschrieben, Blickrichtung planende Person

- Person steuert den Plan und die Aktivitäten

- Zielrichtung: Stärkung und Verwirklichung der Ziele des Planenden durch das Angebot geeigneter individueller Maßnahmen, lernende Organisation

Quelle: »I want my dream! « Persönliche Zukunftsplanung. Neue Perspektiven und Methoden einer individuellen Hilfeplanung mit Menschen mit Behinderungen. Autor: Stefan Doose.

Internet http://bidok.uibk.ac.at/library/doose-zukunftsplanung.html 
für die deutsche Eingliederungshilfe aus dem Westen wehen? Welche Möglichkeiten bestehen, um die aufgezeigten Defizite bei den Planungen individueller Hilfeleistungen zu reduzieren?

Zunächst hätte ein von der Selbstvertretung von Menschen mit Behinderung entwickeltes Instrument zur individuellen Hilfeplanung per se einen emanzipatorischen Charakter und seine Handhabbarkeit und Benutzerfreundlichkeit verstünden sich von selbst. Des Weiteren würde eine derartige Planung dem entgegenwirken, dass die Planungen von den Interessen von Leistungserbringern oder Leistungsträgern dominiert sind. Last, but not least könnte ein von der
Selbstvertretung entwickeltes Planungsinstrument - entsprechende Bildungsund Sensibilisierungsmaßnahmen für die beteiligten professionellen Akteure vorausgesetzt - eine inklusive Dynamik in die individuellen Teilhabeplanungen bringen, so dass Ressourcen aktiviert und im Sinne des Beschlusses der Arbeits- und Sozialministerkonferenz (ASMK) von 2009 »Handlungsstrategien zum Auf- und Ausbau eines inklusiven Sozialraumes « (ASMK 2009: 9) erarbeitet werden könnten.

\section{Literatur}

Arbeits- und Sozialministerkonferenz (ASMK) (2009): Beschlussprotokoll der 86. Konferenz der Ministerinnen und Minister, Senatorinnen und Senatoren für Arbeit und Soziales der Länder am 25./26. November 2009 in Berchtesgaden. Internet http://www.alle-inklusive.de/wp-content/uploads/2009/12/ergebnis-asmk2009.pdf, zuletzt geprüft am 02.09.2011.

Bundesministerium für Arbeit und Soziales (BMAS) (2011): Nationaler Aktionsplan der Bundesregierung zur Umsetzung des Übereinkommens der Vereinten Nationen über die Rechte von Menschen mit Behinderungen. Internet http://www.53gradnord.com/fileadmin/dokumente/Materialien/Aktionsplan_Umsetzung_UN-Konvention.pdf, zuletzt geprüft am 02.09.2011.

Bäcker, Gerhard; Nägele, Gerhard; Bispinck, Reinhard; Hofemann, Klaus; Neubauer, Jennifer (2008): Sozialpolitik und soziale Lage in Deutschland. Wiesbaden: VS Verlag für Sozialwissenschaften.

Evers, Adalbert (2011): Wohlfahrtsmix und soziale Dienste. In: Evers, Adalbert; Heinze, Rolf G.; Olk, Thomas (Hg.): Handbuch Soziale Dienste. Wiesbaden: VS Verlag für Sozialwissenschaften, S. 265-283.

Frevel, Bernhard; Dietz, Berthold (2008): Sozialpolitik kompakt. 2. Auflage. Wiesbaden: VS Verlag für Sozialwissenschaften.

Kunze, Heinrich; Kronenberger, Gerhard; Krüger, Ulrich; Schönhut-Keil, Evelin (Hg.) (2008): Der Reiz des Unentdeckten. Neue Wege zu personenzentrierten Teilhabeleistungen in Hessen. Bonn: Psychiatrie-Verlag.

Offe, Claus (2002): Staat, Markt und Gemeinschaft. Gestaltungsoptionen im Spannungsfeld dreier politischer Ordnungsprinzipien. In: Friedrich Ebert Stiftung (Hg.): Die Bürgergesellschaft. Perspektiven für Bürgerbeteiligung und Bürgerkommunikation. Bonn: Dietz-Verlag, S. 65-84.

Rohrmann, Albrecht; Barth, Cordula; Schädler, Johannes; Althaus, Nadja (2011): Auswertung Anonymisierte ITP. Internet http://www.uni-siegen.de/zpe/projekte/aktuelle/perseh/dokumente_downloads.html?lang=de, zuletzt geprüft am 02.09.2011.

Rohrmann, Albrecht; Althaus, Nadja; Barth, Cordula; Schädler, Johannes (2011a): Rekonstruktion individueller Teilhabearrangements. Internet http://www.uni-siegen.de/zpe/projekte/aktuelle/perseh/dokumente_downloads.html?lang=de, zuletzt geprüft am 02.09.2011.

Rohrmann, Albrecht; Schädler, Johannes; Althaus, Nadja; Barth, Cordula (2011b): Auswertung der schriftlichen Befragung der Leistungsberechtigten. Internet http://www.uni-siegen.de/zpe/projekte/aktuelle/perseh/dokumente_downloads.html? lang=de, zuletzt geprüft am 02.09.2011.

\section{Soziale Selbsthilfe}

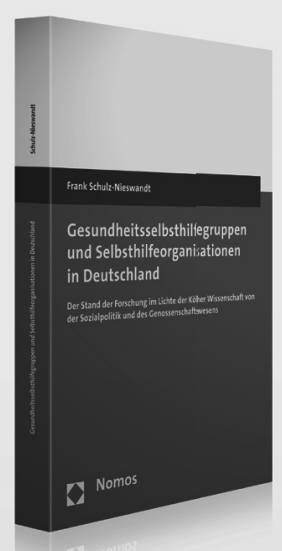

Gesundheitsselbsthilfegruppen und Selbsthilfeorganisationen in Deutschland

Der Stand der Forschung im Lichte der Kölner Wissenschaft von der Sozialpolitik und des Genossenschaftswesens Von Prof. Dr. Frank SchulzNieswandt

2011, 76 S., brosch., 19,- $€$ ISBN 978-3-8329-7101-4

Als Teil des bürgerschaftlichen Engagements und des Dritten Sektors sind soziale Gesundheitsselbsthilfegruppen wenig erforscht. Das Buch gibt einen Überblick über den Forschungsstand und definiert den weiteren Forschungsbedarf. Soziale Selbsthilfe wird genossenschaftsartig als selbstorganisierte Hilfe auf der Basis der Gegenseitigkeit verstanden.

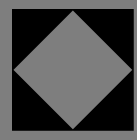
Nomos 